\title{
Trois fragments hittites inédits en provenance d'une collection
} privée d'Istanbul

Alice Mouton

\section{Citer ce document / Cite this document :}

Mouton Alice. Trois fragments hittites inédits en provenance d'une collection privée d'Istanbul. In: Anatolia Antiqua, Tome 20, 2012. pp. 13-17;

doi : 10.3406/anata.2012.1321

http://www.persee.fr/doc/anata_1018-1946_2012_num_20_1_1321

Document généré le 15/05/2016 


\section{Alice MOUTON ${ }^{*}$}

\section{TROIS FRAGMENTS HITTITES INEDITS EN PROVENANCE D'UNE COLLECTION PRIVEE D'ISTANBUL ${ }^{1}$}

La collection Arnas d'Istanbul, par ailleurs très riche en artefacts des époques classiques, comporte trois fragments de tablettes hittites. Ces fragments ont été acquis par M. A. N. Arnas et sont enregistrés à la Direction des Musées archéologiques d'Istanbul. Mes remerciements vont à M. A. N. Arnas qui m'a officiellement autorisée à examiner et publier ces fragments, ainsi qu'à $M^{\text {clle }} \mathrm{G}$. Mümin qui m'a fourni les photographies des tablettes et m'a ouvert les portes de la collection privée en septembre 2007 pour que je puisse collationner les documents originaux. Je souhaite également remercier le Pr. T. Drew-Bear qui m'a mise en contact avec M $^{\text {elle }}$ G. Mümin. Enfin, je suis reconnaissante envers les Dr. M. Doğan-Alparslan et M. Alparslan, ainsi qu'envers $M^{\text {me }} M$. Çifçi qui m'ont aidée à obtenir les autorisations nécessaires à la publication de cet article.

Les deux premiers fragments, 1508 (a) et 1508 (b), pourraient appartenir au corpus de la fête du KI.LAM étant donné que les divinités U.GUR et Zuliya (une divinité-fleuve), d'une part, et les hommes-loups, d'autre part, sont mentionnés. Cette fête d'origine hattie semble être la seule à rassembler tous les éléments présents dans ces deux fragments ${ }^{2}$. En revanche, le troisième fragment, 1508 (c), attestant la divinité Lullayama, se rapproche davantage des fêtes de l'AN.TAH.ŠUM et de la hâte (nuntarriyašhaš).

\subsection{8 (a) (Fig. 1 et 2)}

Fragment en langue vieil hittite d'une fête religieuse mentionnant le roi et le chef de ses gardes du corps $^{3}$, ainsi que la prêtresse AMA.DINGIR ${ }^{L I M 4}$. On y fait également allusion aux divinités Halki, Zuliya et U.GUR ${ }^{5}$. Ces divers éléments se retrouvant ensemble dans la fête du KI.LAM, il est possible que ce fragment provienne de cette composition ${ }^{6}$. Le fragment ne fournit que peu d'éléments de paléographie. On remarquera toutefois les formes "récentes" des signes $\mathrm{ZU} * \pi, \mathrm{DA} \approx \mathrm{LI} * \mathrm{k}$ et $\mathrm{HA} \approx<$. Fort de ces observations, il est possible de dater ce fragment de la fin de l'époque impériale (OH/LNS).

$$
\begin{aligned}
& \text { col. de gauche } \\
& \text { x+3. [..]x-ni } \\
& 4,[\ldots] \\
& 5,[\ldots] \mathrm{x} \\
& 6,[\ldots]{ }^{\mathrm{D} U} \mathrm{G} \cdot \mathrm{GUR} \\
& 7,[\ldots] \mathrm{x}-\mathrm{in}^{7}
\end{aligned}
$$

*) CNRS Strasbourg,

1) Les abréviations employées ici sont celles se trouvant dans : H. G. Güterbock et H. A. Hoffner (éd.), The Hittite Dictionary of the Oriental Institute of the University of Chicago (= CHD), L-N, Chicago 1989 : XXI-XXIX; CHD P, Chicago 1997 : VII-XXVI ; CHD Š, Chicago 2002 : VI-VIII.

2) Pour ne pas alourdir cet article, je ne donne pas la liste de toutes les fêtes religieuses hatties (CTH 591 à 685). Le lecteur pourra se reporter au CTH en ligne du site du Hethiter Portal Mainz (http://www.hethport.uni-wuerzburg.de/CTH/).

3) Pour les attestations du chef des gardes du corps du roi en contexte de fêtes religieuses, voir Pecchioli Daddi 1982 : $549-552$.

4) Pour cette prêtresse, voir Pecchioli Daddi $1982: 408-412$ et, en dernier lieu, Taggar-Cohen $2006: 30-31$.

5) Pour les attestations de ces trois divinités, voir van Gessel $1998: 72-76,600-601$ et 837-840 respectivement.

6) Après avoir mené l'enquête sur les autres grandes fêtes hatties, il s'avère, en effet, que seule la fête du KI.LAM rassemble tous les éléments mentionnés ici. Ainsi, par exemple, la fête de l'AN.TAH.ŠUM ne semble pas mentionner Zuliya (d'après van Gessel 1998 : 600-601), la prêtresse AMA.DINGIR et Halki paraissent absentes de la fête du mois (d'après Klinger 1996), même chose pour la fête de la hâte (d'après Nakamura 2002), etc.

7) Peut-être ${ }^{D}$ Wašizzalin, comme dans la fête du KI.LAM (Singer $1984: 65$ ). 


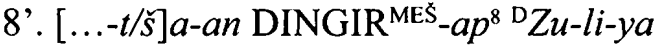 \\ 9'. [...(-)t]a(-)x-pu-na

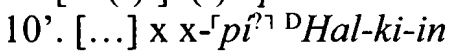 \\ col. de droite \\ $\mathrm{x}+1 .\left\ulcorner n u^{\urcorner}{ }^{\top} \mathrm{AMA}{ }^{\urcorner}\right.$.DINGIR $[\ldots]$
}

2'. GAL $M E-\check{S} E-D[I \ldots]$

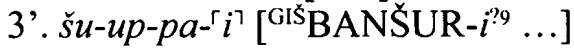

4'. da-a-i[..]

5'. NINDA $h a-{ }^{-} a^{7}-l\left[i^{?}-i n^{? 10} \ldots\right]$

6'. ta-at $[\ldots]$

7'. LUGAL-uš [...]

8'. 'LU'?? x x[...]

9'. Ú-U[L ...]

10'. LUGAL-ǔ̌ [...]

11'. GIŠ INANNA.[GAL/TUR ...]

12'. 1 NINDA? $x[\ldots]$

13'. LUGAL-uš [...]

14'.e-ku-zi [...]

15'. 1 NINDA.GUR 4 .RA $1[\ldots]$

16'. GIŠ-ru I $\check{S}-T\left[U^{?}{ }^{\prime} \ldots\right]$

\section{1508 (b) (Fig. 3 et 4)}

Fragment en langue vieil hittite d'une fête religieuse mentionnant le couple royal, les hommesloups, un prêtre et des lyres. Les hommes-loups se retrouvent dans plusieurs fêtes religieuses d'origine hattie, dont les fêtes de l'AN.TAH.ŠUM et du KI.LAM ${ }^{11}$. Ce fragment contient encore moins de "signes diagnostics" que le précédent, ce qui en rend la datation incertaine. Seule la forme "récente" du signe HA , forme utilisée deux fois dans le fragment, tendrait à dater ce manuscrit de la fin de l'époque impériale, à l'instar de 1508 (a). Si ce

fragment doit bien être daté $\mathrm{OH} / \mathrm{LNS}$ et étant donné que ses éléments constitutifs rappellent, entre autres compositions, la fête du KI.LAM, tout comme 1508 (a), il serait tentant de penser que 1508 (a) et 1508 (b) sont deux fragments de la même tablette, sans joint direct. En effet, ils semblent avoir été collectés en même temps ${ }^{12}$. Toutefois, en confrontant la forme des signes des deux fragments, on remarque des différences notables, en particulier pour les signes INANNA et TA (voir tableau en annexe). Il faut donc se résoudre à les considérer comme provenant de deux tablettes distinctes.

\begin{tabular}{l} 
col. de gauche \\
$\mathrm{x}+2 .[\ldots-a] z^{?}$ \\
$3^{\prime} \cdot[\ldots] \mathrm{x}-z a$ \\
$4^{\prime} \cdot[\ldots] 6^{?}$ \\
\hline $5^{\prime} \cdot[\ldots]-r i-i \check{s}$ \\
$6^{\prime} \cdot\left[\ldots \mathrm{URU} A^{\prime}-r\right] i-i n-n a$ \\
$7^{\prime} \cdot[\ldots] \mathrm{x}-i \check{S}^{-}$
\end{tabular}

col. de droite

$$
\begin{aligned}
& \mathrm{x}+1 . \mathrm{x} \times[\ldots] \\
& \text { 2'. na-at } \mathrm{E}[\mathrm{GIR} ? . . .]
\end{aligned}
$$

\section{3'. LUGAL MUNUS.LUGAL- $y[a \ldots]$ \\ 4'. GIŠ DINANNA.GAL x[...]}

$$
\begin{aligned}
& \text { 5'. LU' }{ }^{M E S ̌ ~ U R . B A R . R[A ~ . . .] ~} \\
& \text { 6'. ha-aš-ša-an-[...] } \\
& \text { 7'. } n u \text { LÚ }{ }^{\mathrm{MESS}} \mathrm{U} \text { [R.BAR.RA ...] } \\
& \text { 8'. wa-ra-aš-ha-[... } \left.{ }^{13}\right] \\
& \text { 9'. UGULA LÚ }{ }^{\mathrm{MES}} \mathrm{x}[\ldots] \\
& \text { 10'. ta } A-N A \times[\ldots] \\
& 11 \text { '. } 1^{\mathrm{DUG}} h u-u\left[p^{?}-p a r^{?}{ }^{14} \ldots\right]
\end{aligned}
$$

12'. LÚSANGA [...]

13'. $t a-a \check{s}-s ̌[i \ldots]$

8) La lecture du signe est assurée par collation du fragment original. Bien que la forme hattie wašhap pour "dieux" soit attestée dans plusieurs textes relatifs à des fêtes religieuses (Soysal $2004: 959)$, il est sans doute préférable de penser que DINGIR" correspond, dans notre fragment, à DINGIR ${ }^{\text {MES }}$ auquel s'ajoute la particule locale enclitique - $(a)$ pa, car l'environnement linguistique de cet ensemble paraît hittite, et non hatti. La forme -ap pour-(a)pa est attestée dans plusieurs textes en langue vieil hittite (Hoffner et Melchert $2008: 378$ et $\mathrm{HW}^{2} \mathrm{~A}, 125-130$ ).

9) Proposition personnelle de restauration à partir de parallèles connus dans les fêtes du KI.LAM et de la hâte

10) Type de pain (Hoffner $1974: 153-154$ ) ou bien NINDA häliš, où hāli- signifie "portion" (HED H, 23-24) ? Fréquemment mentionné dans le corpus de la fête du KI.LAM (Singer 1984 : 132), ainsi que dans plusieurs autres fêtes religieuses de l'époque vieil hittite (Neu $1983: 43-44$ ).

11) Pecchioli Daddi $1982: 373-375$.

12) D'après une information fournie oralement par $M^{\text {elle }}$ Mümin.

13) Voir wa-ar-aš-hu-u-wa-ar dans KBo 10.28+ i 4' appartenant au corpus de la fête du KI.LAM, wa-ar-ăs-hu-ar dans KBo $60.218 \mathrm{Ro}^{\text {? }} 3$ ' qui décrit une fête religieuse de la sphère hattie, et $\dot{u}$-ar-aš-ha-an-zi dans KUB 10.66 vi 4 décrivant également une fête religieuse (Singer $1983: 84$ note 70 et Kloekhorst $2008: 959-960$ ).

14) Type de récipient mentionné dans le corpus de la fête de la hâte (Nakamura $2002: 338$ ). 


\section{1508 (c) (Fig. 5 et 6 )}

Fragment de fête religieuse mentionnant la divinité Lullayama, la grande assemblée et des lyres. Lullayama est également attestée dans plusieurs fêtes d'origine hattie, telles que la fête de l'AN.TAH.ŠUM et la fête nuntarriyašhaš ${ }^{15}$. Notons qu'elle ne semble pas l'être dans la fête du KI.LAM ${ }^{16}$. Le fragment est trop petit pour que l'on puisse en donner une datation, aussi bien au niveau de la langue que du ductus. Seule la forme "récente" du signe LI est bien reconnaissable, malgré la lacune dans sa partie basse.

\begin{tabular}{|c|}
\hline 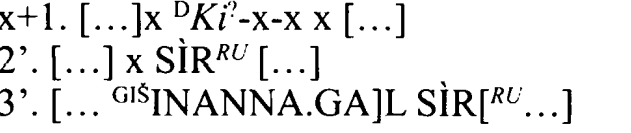 \\
\hline 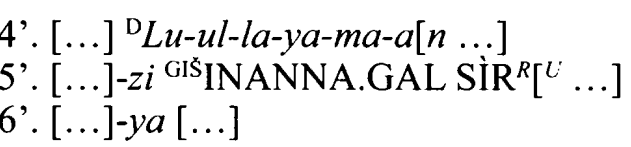 \\
\hline
\end{tabular}

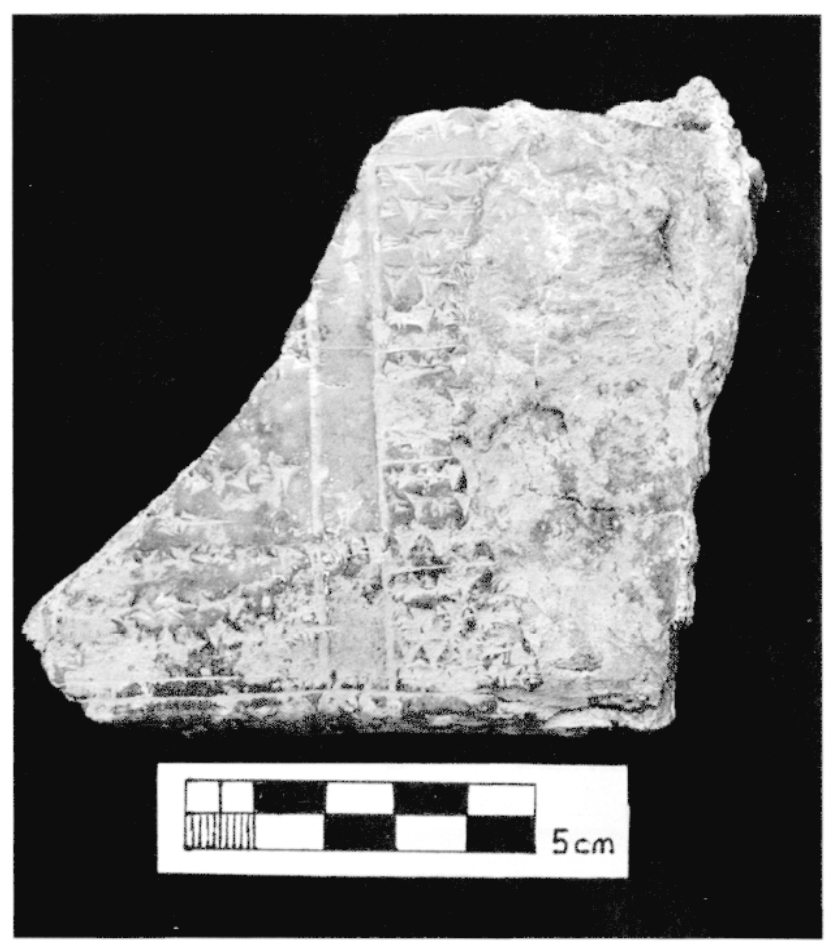

Fig. 1 : Photographie de 1508 (a) (G. Mümin).

$$
\begin{aligned}
& \text { 7'. [... šal]-li a-še-eš-na-aš [...] } \\
& 8 \text { '. [...]-zi [..] } \\
& \text { 9'. [...] Ú-UL e-e[šs-zi ...] }
\end{aligned}
$$

A.M.

\begin{tabular}{|c|c|c|}
\hline & $1508(a)$ & $1508(b)$ \\
\hline IN & 全供 & 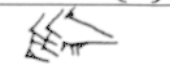 \\
\hline NA & NK & r \\
\hline AT & 송 & 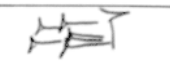 \\
\hline LUGAL & 521 & 级击 \\
\hline INANNA & NFT & $\overline{7}$ \\
\hline AN & ${ }_{N-T}$ & $\mapsto r$ \\
\hline PA & 年 & 7 \\
\hline TA & 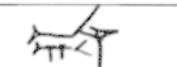 & tres \\
\hline
\end{tabular}
Annexe : Tableau comparatif entre le ductus de 1508 (a) et celui de 1508 (b)

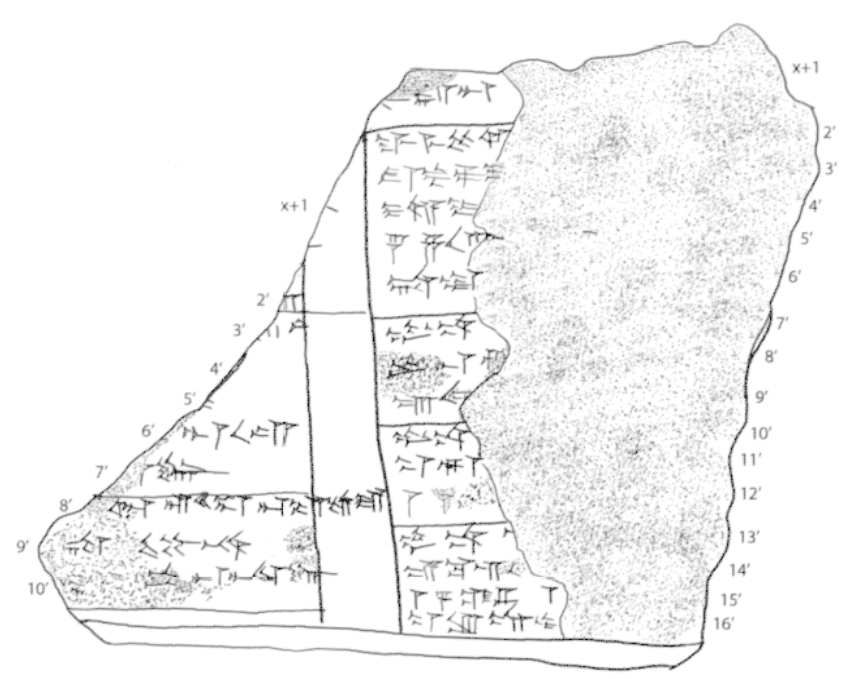

Fig. 2 : Copie de 1508 (a) (A. Mouton). 

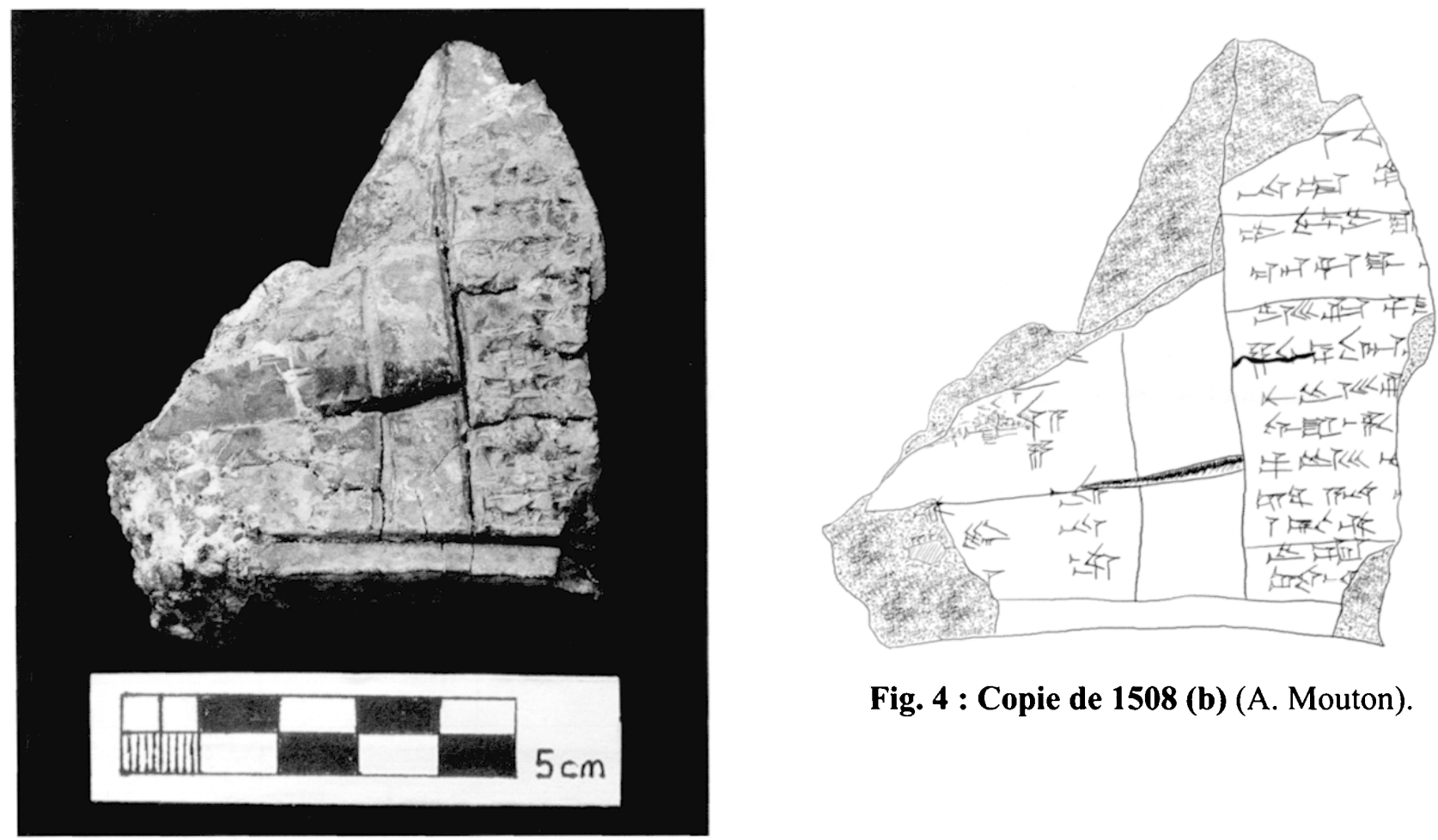

Fig. 4 : Copie de 1508 (b) (A. Mouton).

Fig. 3 : Photographie de 1508 (b) (G. Mümin).
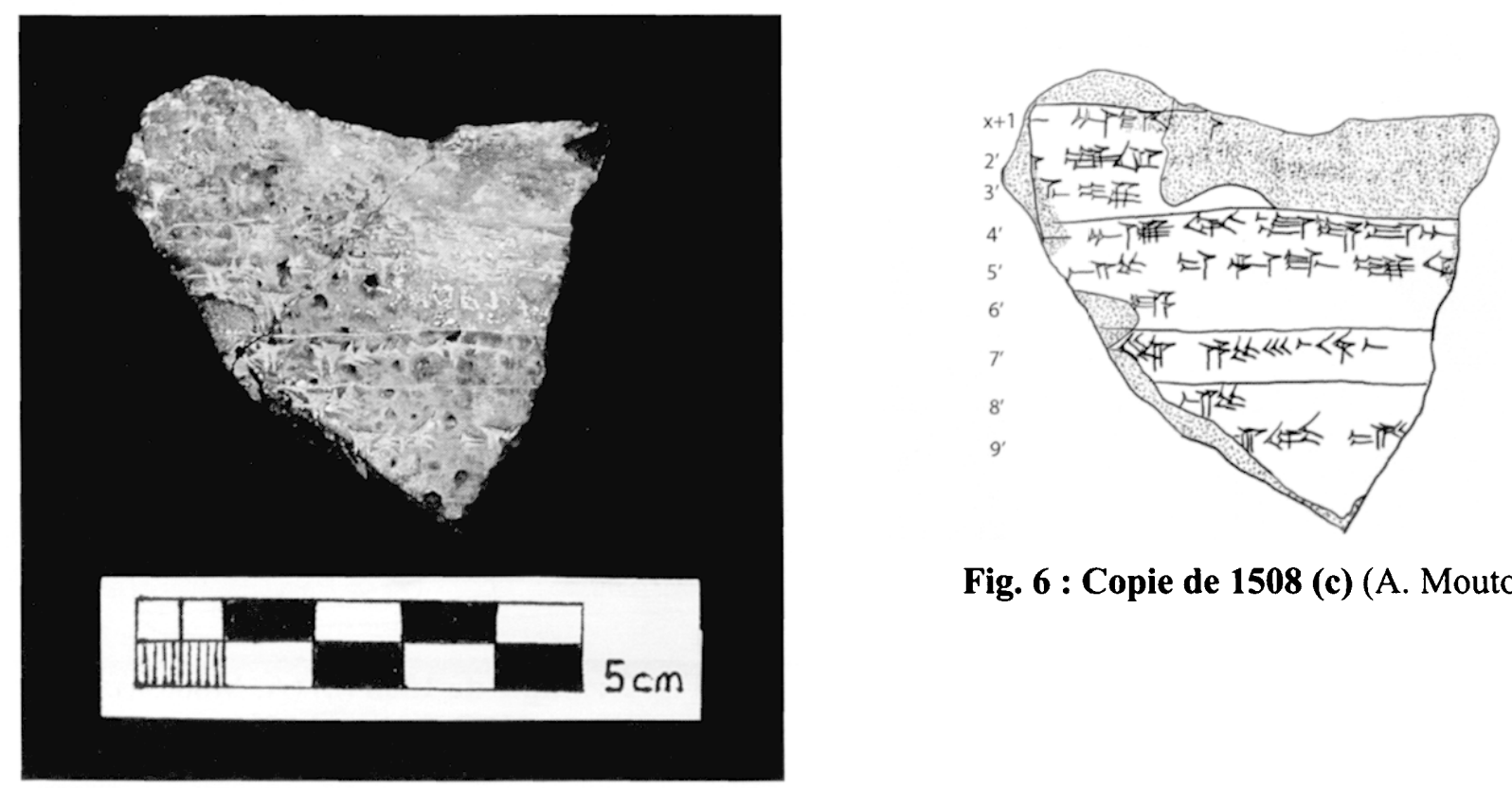

Fig. 6 : Copie de 1508 (c) (A. Mouton).

Fig. 5 : Photographie de 1508 (c) (G. Mümin). 


\section{BIBLIOGRAPHIE}

Hoffner, H. A., 1974 : Alimenta Hethaeorum. Food Production in Hittite Asia Minor, AOS 55, New Haven. Hoffner, H. A. et Melchert, H. C., 2008 : A Grammar of the Hittite Language, Languages of the Ancient Near East 1, Winona Lake.

Klinger, J., 1996 : Untersuchungen zur Rekonstruktion der hattischen Kultschicht, StBoT 37, Wiesbaden.

Kloekhorst, A., 2008 : Etymological Dictionary of the Hittite Inherited Lexicon, Leiden Indo-European Etymological Dictionary Series 5, Leyde-Boston.

Nakamura, M., 2002 : Das hethitische nuntarriyašhaFest, PIHANS 94, Leyde.

Neu, E., 1980 : Althethitische Ritualtexte in Umschrift, StBoT 25, Wiesbaden.

- 1983: Glossar zu den althethitischen Ritualtexten, StBoT 26, Wiesbaden.
Pecchioli Daddi, F., 1982 : Mestieri, professioni e dignità nell'Anatolia ittita, Incunabula Graeca 79, Rome. Popko, M., 1994 : Zippalanda. Ein Kultzentrum im hethitischen Kleinasien, THeth 21, Heidelberg.

Singer, I., 1983 : The Hittite KI.LAM Festival. Part One, StBoT 27, Wiesbaden.

- 1984 : The Hittite KI.LAM Festival. Part Two, StBoT 28, Wiesbaden.

Soysal, O., 2004 : Hattischer Wortschatz in hethitischer Textüberlieferung, HdO I/74, Leyde-Boston.

Taggar-Cohen, A., 2006 : Hittite Priesthood, THeth 26, Heidelberg.

van Gessel, B. H. L., 1998 : Onomasticon of the Hittite Pantheon, HdO I/33, Leyde-New York-Cologne.

Yoshida, D., 1996 : Untersuchungen zu den Sonnengottheiten bei den Hethitern, THeth 22, Heidelberg. 